\title{
Fabrication and Characteristics of Micro-Electro-Mechanical-System-Based Gas Flow Sensor
}

\author{
Ju Chan Choi ${ }^{1}$, June Kyoo Lee ${ }^{1}$, and Seong Ho Kong1,2,+
}

\begin{abstract}
This paper proposes a highly-sensitive gas flow sensor with a simple structure. The sensor is composed of a micro-heater for heating the gas medium and a pair of temperature sensors for detecting temperature differences due to gas flow in a sealed chamber on one axis. Operation of the gas flow sensor depends on the transfer of heat through the air medium.The proposed gas flow sensor has the capability to measure gas flow rates $<5 \mathrm{~cm}^{3} / \mathrm{min}$ with a resolution of approximately $0.01 \mathrm{~cm}^{3} / \mathrm{min}$. Furthermore, this paper reports some additional experiment results, including the sensitivity of the proposed gas flow sensor as a function of operating current and the flow of different types of gas(oxygen, carbon dioxide, and nitrogen). The fabrication process of the proposed sensor is very simple, making it a good candidate for mass production.
\end{abstract}

Keywords : Gas Flow Sensor, Flow Meter, MEMS

\section{INTRODUCTION}

In recent years, great efforts have been devoted to making gas flow sensors for many application fields such as automotive, environmental monitoring, and industrial process control[1]. There have been some reports on welldeveloped thermal mechanism-based flow sensors over the years[2-4]. In addition, many new structures have been introduced to improve performance of the performance of flow sensors[5-8]. There has recently been an increased need for the measurement of very small flow rates in micro-channels for biochips, gas sensors, and industrial applications. Detection of the micro-scale flow is very important for fluid control in microfluidics.

In this paper, a novel highly-sensitive gas flow sensor is proposed by means of temperature sensor optimization. The fabricated flow sensor is evaluated in a flow range of 1 $-5 \mathrm{~cm}^{3} / \mathrm{min}$, and it shows a linear response with a high resolution of $0.01 \mathrm{~cm}^{3} / \mathrm{min}$ in the operating range.

\footnotetext{
${ }^{1}$ Graduate School of Electrical Engineering and Computer Science, IT3-213A, Kyungpook National University

${ }^{2}$ School of Electronics Engineering, College of IT Engineering,

IT3-213, Kyungpook National University

+Corresponding author: shkong@knu.ac.kr

(Received : Jun. 27, 2011, Revised: Aug. 18, 2011. Accepted: Sep. 7, 2011)
}

\section{DESIGN}

\subsection{Material for temperature sensing}

To fabricate a highly-sensitive gas flow sensor, an optimization of temperature sensor is very important because the performance of thermal gas flow sensor is determined by the sensitivity of temperature sensor. The temperature coefficient of resistance(TCR) value is one of the figures of merit that influence the performance of temperature sensor, so selecting a material with a high TCR value is very critical.

Table 1 shows the temperature coefficient of resistivity for a variety of materials. Platinum has an anti-corrosive property, and its resistance change as a function of temperature is quite linear in a broad range. Nickel has a high TCR value and a low cost compared to other metals. Due to these merits, the proposed flow sensor uses nickel for both the temperature sensor and the heater materials.

Table 1. Temperature coefficient of resistivity for different materials

\begin{tabular}{cc}
\hline \hline Material & $\alpha\left[\left({ }^{\circ} \mathrm{C}\right)-1\right]$ \\
\hline Copper & $4.29 \times 10^{-3}$ \\
Iron & $6.41 \times 10^{-3}$ \\
Nickel & $6.00 \times 10^{-3}$ \\
Platinum & $3.93 \times 10^{-3}$ \\
Chromel & $0.58 \times 10^{-3}$ \\
Nichrome & $0.40 \times 10^{-3}$ \\
\hline \hline
\end{tabular}




\subsection{Sensitivity change by temperature sensor shape}

The sensitivity of the proposed gas flow sensor can be expressed as:

$S_{\text {sens.flow }} \propto S_{\text {sens.temp }} \propto \Delta V_{\text {out.flow }} \propto \Delta R_{\text {change.temp }}$

Where $S_{\text {sens.flow }}$ and $\Delta V_{\text {out.flow }}$ are the sensitivity and output voltage of the proposed gas flow sensor, respectively. $S_{\text {sens.temp }}$ and $\Delta R_{\text {change.temp }}$ are the sensitivity and resistance change of the temperature sensor formed on the proposed gas flow sensor, respectively.

The sensitivity of the proposed gas flow sensor is proportional to the sensitivity of the temperature sensor because the proposed gas flow sensor operates by detecting a resistance change in the temperature sensor. If the sensing current of the temperature sensor is fixed, as resistance of the temperature sensor increase due to Ohm's law, the output voltage of the gas flow sensor increases.

First, titanium is deposited on the silicon membrane to create adhesion between the silicon dioxide and the nickel. A 200-nm-thick nickel temperature sensor is deposited by e-beam evaporation and patterned using a lift-off process. Various temperature sensors have been patterned and measured. The results of the experiment are shown in Figs. 1 and 2. During the experiment, the sensing current for the temperature sensor is fixed at $10 \mathrm{~mA}$.

Fig. 1 shows the sensing characteristics of the sensors with different line lengths. In the patterning of S11 and $\mathrm{S} 15$, the wiresare connected in a series. The resistance change increases as the wire length increases since the moving carrier is likely to be more scattered than the short wire.

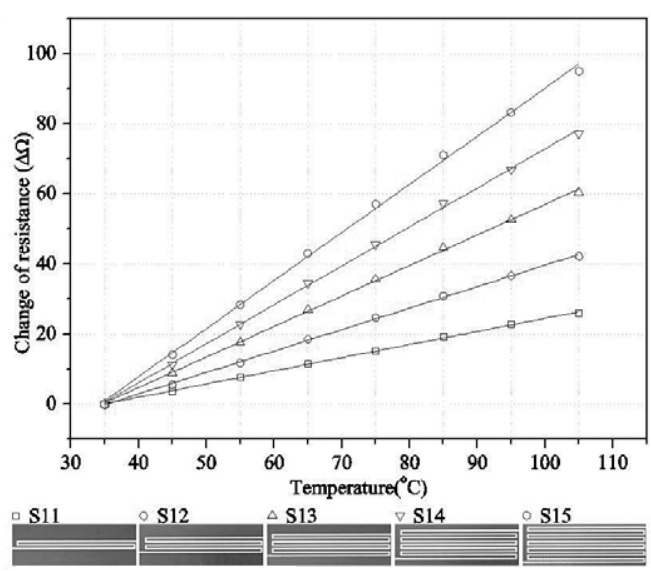

Fig. 1. Resistance changes of the temperature sensor at different line lengths.
Fig. 2 shows the sensing characteristics of the temperature sensors with different pattern widths. The resistance change of the temperature sensor could be expected to decrease with a large pattern width because the carriers pass through the temperature sensor more easily at the same sensing current.

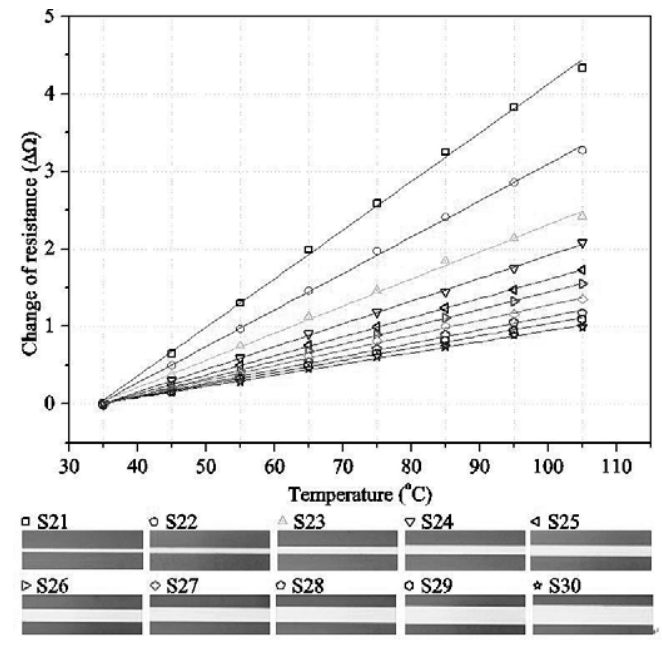

Fig. 2. Resistance changes of the temperature sensor at different widths.

As shown in Figs. 1 and 2, the resistance change is proportional to the length and inversely proportional to the pattern width of the temperature sensor.

\subsection{Gas flow sensor design}

Fig. 3 shows a schematic of the operating principle of the gas flow sensor. The gas flow sensor is based on heat transfer through the gas medium. Therefore, the heated temperature sensor is cooled by a passing flow and the local cooling rate depends on the flow velocity.

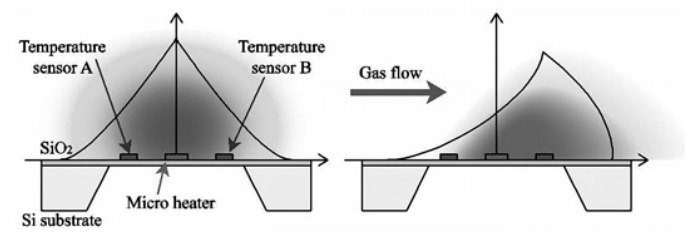

Fig. 3. Operating principle of the gas flow sensor.

Fig. 4 shows the fabricated gas flow sensor, which is composed of a micro-heater for heating the gas medium and four temperature sensors for detecting movement of the gas medium usingdetected temperature differences in 
the sealed cavity. Each component, the micro-heater and the temperature sensor, is formed using nickel $(200 \mathrm{~nm}) /$ titanium( $20 \mathrm{~nm}$ ) on a silicon dioxide membraneto create thermal isolation between the micro-heater and the temperature sensor.
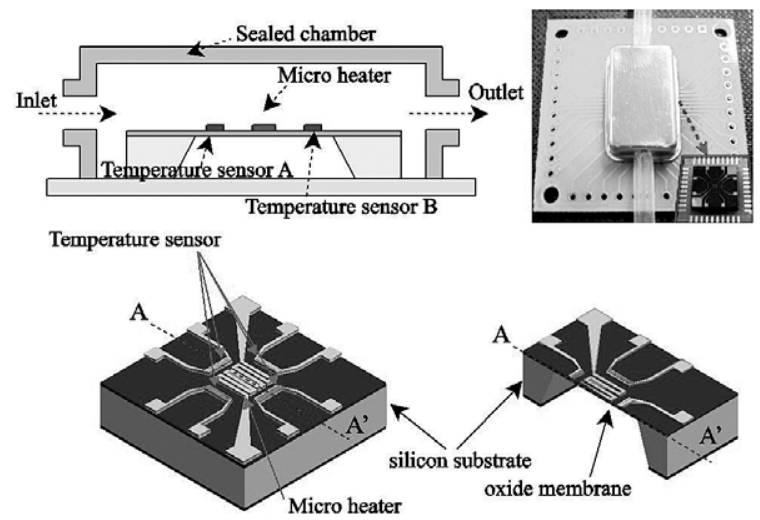

Fig. 4. Fabricated gas flow sensor.

\section{MEASUREMENT}

Fig. 5 shows the measurement setup for measuring gas flow. Measurements are performed using a heating current of $50 \mathrm{~mA}$ and a sensing current of $10 \mathrm{~mA}$, while the gas is controlled via mass flow control(MFC).

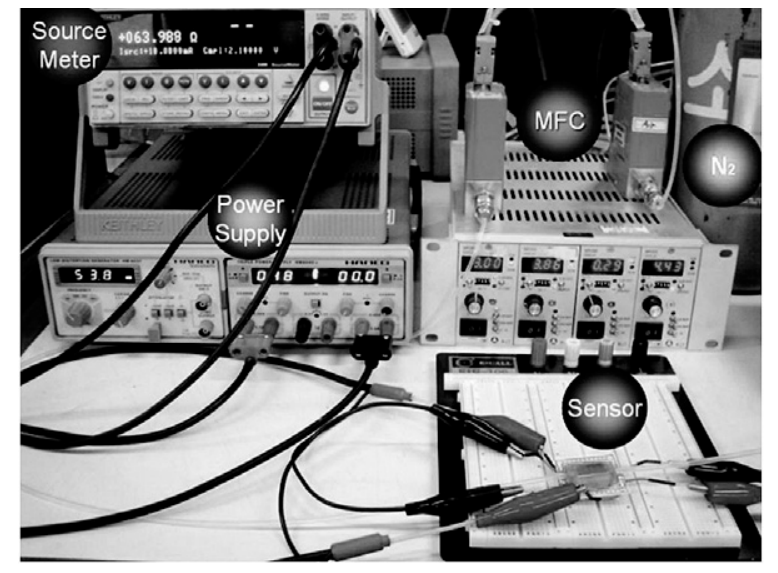

Fig. 5. Measurement system.

Fig. 6 shows standard measured results of the proposed gas flow sensor. The sensor has a $0-5 \mathrm{~cm}^{3} / \mathrm{min}$ detecting range, and flow directions can be distinguished using differential output results of temperature sensors A and B in a line on one axis.

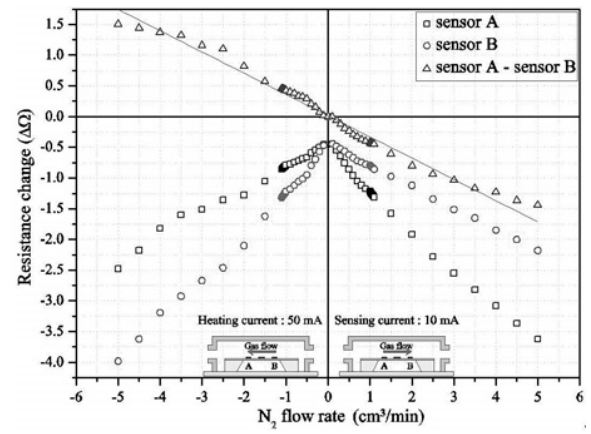

Fig. 6. Characteristics of the fabricated temperature sensor.

Fig. 7 shows the resistance change of the proposed gas flow sensor as a function of the heating current. The flow sensor shows elevated sensitivity as heating current increases.

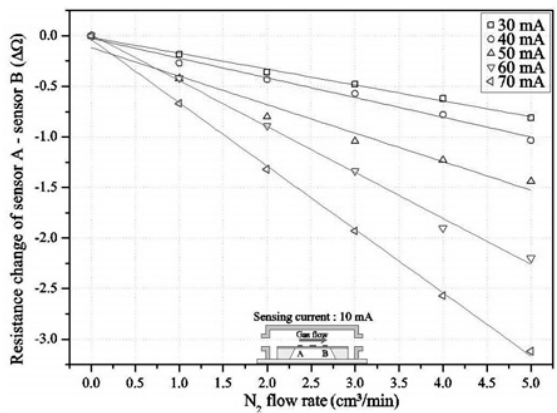

Fig. 7. Output characteristics as a function of heating current.

The measurement results of a small gas flow rate using the proposed sensor are shown in Fig. 8. The fabricated flow sensor has a very high resolution of approximately $0.01 \mathrm{~cm}^{3} / \mathrm{min}$.
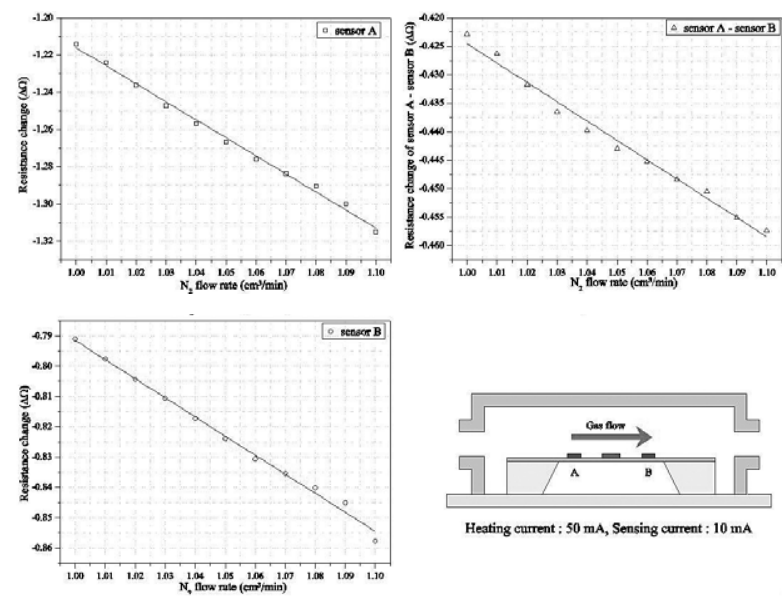

Fig. 8. The resolution of the fabricated gas flow sensor. 
Detection of very small flow in the micro-channel is an important sensing technique for microfluidics, microanalysis, biochip, and microchemistry. The proposed sensor is a good highly sensitive flow sensor candidate due to its high sensitivity and simple structure.

Fig. 9 shows the output characteristics as a function of gas type. The proposed sensor shows different sensitivities to the different gases due to their distinct thermal properties.

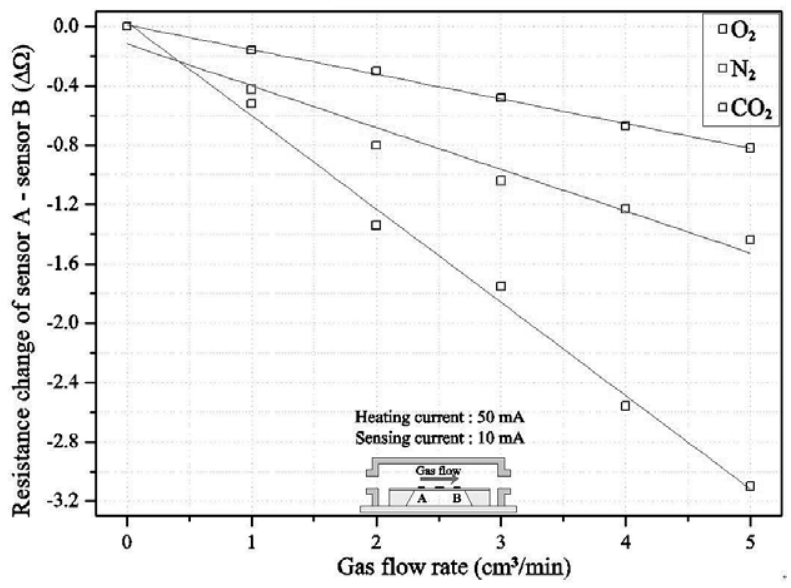

Fig. 9. Output characteristics of the different gas types.

\section{DISCUSSION}

Sensitivity improvement of a thermal gas flow sensor by simply modifying the temperature sensor shape has many advantages. The sensitivity is proportional to the length and inversely proportional to the cross-section of the temperature sensor.

Therefore, an increased length and decreased crosssection of the temperature sensor directly improve thermal sensitivity. Infinitely extending the length of the temperature sensor to create superior sensitivity, however, is impractical and demerits the sensor's potential application in micro-flow sensing systems.

The one way to solve this problem is to reduce the thickness of the temperature sensor. Decreased thickness of the temperature sensor leads to a decreased cross-section and increases sensor sensitivity, whereas reduced thickness of the temperature sensor partially compromises the sensor footprint. The patterning method is a quite simple technique for controlling temperature sensor sensitivity without the need for additional processes.

\section{CONCLUSIONS}

A micro-electro-mechanical-systems-based gas flow sensor was proposed and realized using a simple fabrication process. Thin-film micro-heater and temperature sensors are placed on top of silicon dioxide membrane in the line of a flow channel to restrict the heat conduction to the substrate. The sensitivity of the gas flow sensor is improved by simple temperature sensor patterning. The response characteristics in the $0-5$ $\mathrm{cm}^{3} / \mathrm{min}$ flow range were investigated for threedifferent gases, $\mathrm{O}_{2}, \mathrm{~N}_{2}$ and $\mathrm{CO}_{2}$. The proposed sensor could detect various gas flow rates with a resolution of $0.01 \mathrm{~cm}^{3} / \mathrm{min}$.

\section{ACKNOWLEDGMENT}

This work was supported by a National Research Foundation of Korea Grant funded by the Korean government (No. 2010-0001884).

\section{REFERENCES}

[1] P. Bruschi, M. Piotto, and G. Barillaro, "Effects of gas type on the sensitivity and transition pressure of integrated thermal flow sensors", Sens. Actuators A, vol. 132, no. 1, pp. 182-187, 2006.

[2] A. Kularni, S. Patil, R. N. Karekar, and R. C. Aiyer, "Fabrication and characterization of innovative gas flow sensor", Sens. Actuators A, vol. 122, no. 2, pp. 231-234, 2005.

[3] G. Kaltsas and A. G. Nassiopoulou, "Gas flow meter for application in medical equipment for respiratory control: study of the housing", Sens. Actuators A, vol. 110, no. 1-3, pp. 413-422.

[4] H. Ernst, A. Jachimowicz, and G. A. Urban, "High resolution flow characterization in Bio-MEMS", Sens. Actuators A, vol. 100, no. 1, pp. 54-62, 2002.

[5] A. Kunimoto, N. Abe, H. Uchida, and T. Katsube, "Highly sensitive semiconductor NO gas sensor operating at room", Sens. Actuators B, vol. 65, no. 1-3, pp. 122-124, 2000.

[6] N. C. Popa, I. Potencz, and L. Vekas, "Magnetic fluid flow meter for gases", Magnetics, vol. 30, no. 2, pp. 936-938, 1994.

[7] S. G. Joshi, "Surface-acoustic-wave flow sensor", IEEE 
Trans. Ultrason. Ferroelectr., vol. 38, no. 2, pp. 148154, 1991

[8] C. T. Chiang and Y. C. Huang, "A semicylindrical capacitive sensor with interface circuit used for flow rate measurement", IEEE Sens. J., vol. 6, no. 6, pp. 1564-1570.

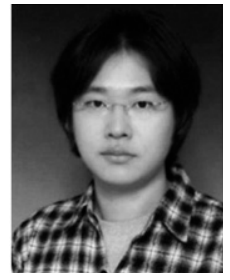

Ju Chan Choi received his B.S. and M.Sc. degrees in electrical engineering and computer science from Kyungpook National University, Korea, in 2007 and 2009 , respectively. He is currently working toward a Ph.D. degree in Electrical Engineering and Computer Science, Kyungpook National University, where he is also working on microsensor and power MEMS. His research interests include MEMS-based sensors and power MEMS fields.

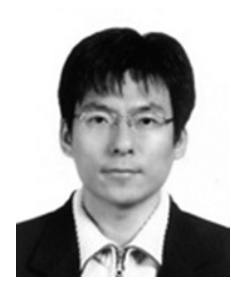

June Kyoo Lee received B.S. and M.S. degrees in Metallurgical Engineering and Sensor Engineering from Kyungpook National University, Korea, in 2001 and 2003, respectively. In 2008, he joined the Sensors \& Actuators Laboratory in the School of Electrical Engineering and Computer Science at Kyungpook National University, where he is currently working as a $\mathrm{Ph} . \mathrm{D}$ candidate. His research interests include electrowetting physics, MEMS-based sensors and actuators, and MEMSbased energy harvesting devices.

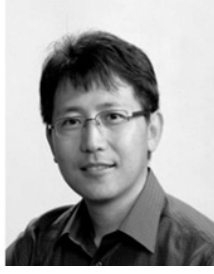

Seong Ho Kong received his B.S. degree in electronic engineering from Kyungpook National University, Korea, in 1993 and his M.Sc. degree in mechatronics and precision engineering from Tohoku University, Japan, in 1996. In 2002, he received his Ph.D. degree in electrical engineering from Delft University of Technology, the Netherlands, with his thesis work concerning infrared micro-spectrometers, and joined ASM Europe as a senior process engineer. In 2004, he joined the School of Electrical Engineering and Computer Science, Kyungpook National University, Korea. His main research activities currently involve the design and fabrication of sensors/actuators based on MEMS technology. 